\title{
Pitch and Duration Pattern Sequence Tests in 7- to 11-Year-Old Children: Results Depend on Response Mode
}

DOI: $10.3766 /$ jaaa.16132

\author{
Sheila Andreoli Balen* \\ David R. Moore $\dagger$ \\ Koichi Sameshimał
}

\begin{abstract}
Background: Pitch pattern sequence (PPS) and duration pattern sequence (DPS) tests are frequently used in the assessment of auditory processing disorder. Current recommendations suggest alternate, interchangeable modes for responding to stimuli.
\end{abstract}

Purpose: The objective of the study is to evaluate the influence of response mode (i.e., humming, pointing, and labeling) and age on PPS and DPS performance of 7- to 11-year-old children.

Research Design: Laboratory-based testing of school children. Cross-sectional comparison of age, with repeated measures of age, test, ear, and response mode.

Study Sample: From 452 children recruited, 228 right-handed children (109 girls) aged 7 years to 11 years 11 months (mean age 9 years 4 months) completed at least one test (PPS: 211, DPS: 198), and 181 children completed both tests. Audiology inclusion criteria include normal hearing thresholds ( $\leq 15 \mathrm{~dB} \mathrm{HL}$ at octave frequencies $250-8000 \mathrm{~Hz}$ ); word recognition in quiet $\geq 92 \%$; tympanogram peak compensated static acoustic compliance 0.4-1.6 mmhos; and tympanometric peak pressure -100 to + $50 \mathrm{daPa}$, all in both ears. Other inclusion criteria were Portuguese as first language; right handed; no musical training; no related, known, or observed phonological, learning, neurologic, psychiatric, or behavioral disorder; otologic history; and delayed neuropsychomotor or language development.

Data Collection and Analysis: PPS: 30 trials per ear and response condition of three consecutive 500 msec duration intermixed high $(1430 \mathrm{~Hz})$ or low $(880 \mathrm{~Hz})$ frequency tones presented monaurally at $50 \mathrm{~dB}$ HL. The first response condition was humming followed by labeling (naming: high or low). DPS: As per PPS except $1000 \mathrm{~Hz}$ tones of intermixed 500 (long) and $250 \mathrm{msec}$ (short) duration. First response was pointing (at a symbolic object) followed by labeling. Trends across age and between tests were assessed using repeated measures generalized linear mixed models. Correlation coefficients were calculated to assess relations among test scores. The two-sided significance level was 0.05 .

Results: Older children performed better than younger children in all tasks. Humming the tone pattern (PPS humming) produced generally better performance than either articulating the attributes of the tones (labeling) or pointing to objects representing tone duration. PPS humming produced ceiling performance for many children of all ages. For both labeling tasks and DPS pointing, performance was better on the PPS than on the DPS, for stimulation of the right than the left ear, and in boys than girls. Individual performance on the two tasks was highly correlated.

Conclusions: Response mode does matter in the PPS and DPS. Results from humming should not be combined with or be a substitute for results obtained from a labeling response. Tasks that rely on labeling a tonal stimulus should be avoided in testing hearing in children or other special populations.

Key Words: auditory processing disorder, children, duration pattern test, pitch pattern test

*Department of Speech, Language and Hearing, Federal University, Rio Grande do Norte, Brazil; †Communication Sciences Research Center, Cincinnati Children's Hospital Medical Center, and Department of Otolaryngology, University of Cincinnati College of Medicine, Cincinnati, OH 45229; \$Department of Radiology and Oncology, School of Medicine, University of São Paulo, São Paulo, Brazil

Corresponding author: Sheila Andreoli Balen, Department of Speech, Language and Hearing, Federal University, Tirol, Natal, Rio Grande do Norte, Brazil 59015-110; Email: sheila@sheilabalen.com.br 


\begin{abstract}
Abbreviations: $\mathrm{AP}=$ auditory processing; $\mathrm{APD}=$ auditory processing disorder; $\mathrm{ASHA}=\mathrm{American}$ Speech-Language-Hearing Association; DL = DPS labeling; DP = DPS pointing; DPS = duration pattern sequence; FPT = frequency pattern test; GLMM = generalized linear mixed models; PPS = pitch pattern sequence
\end{abstract}

\section{INTRODUCTION}

$\mathrm{C}$ hildren attending audiology clinics with reports of listening difficulties who are found to have normal audiograms may be evaluated for an auditory processing disorder (APD). The evaluation typically includes tests for auditory pattern recognition and temporal processing (ASHA, 2005; AAA, 2010). Among the most common of these are variants of the pitch pattern sequence (PPS) and duration pattern sequence (DPS) tests (Emanuel et al, 2011), consisting of sequences of three tones, one of which differs in frequency or duration from the others (Musiek, 1994). Originally developed as tests for the auditory consequences of brain lesions (Musiek and Pinheiro, 1987; Musiek et al, 1990), PPS and DPS tests have been categorized as tests of temporal processing (McDermott et al, 2016). However, the long base duration of the tones (e.g., $500 \mathrm{msec}$ ), the easily discriminable frequency (e.g., 0.5 octaves) and duration (e.g., $250 \mathrm{msec}$ ) differences, and the task requirement to reproduce the correct ordering of the tones make them more appropriately called tests of auditory pattern perception. The perception of auditory patterns is influenced by the acoustic properties of the stimulus and by attention, working memory, and experience (Bregman, 1990; Alain and Woods, 1997). Experience includes formal instruction (e.g., music lessons, Kraus and Chandrasekaran, 2010), statistical learning (Skoe et al, 2015), and informal sources (e.g., language exposure, Zhang et al, 2005; reading, Walker et al, 2006).

Performance of children on the PPS and DPS tests is strongly affected by the order, timing, and number of stimuli, as well as how the sequences are presented (e.g., continuous, discontinuous) and the type of response required (Shin, 2003). Regarding the response, a current clinical recommendation and practice (Musiek, 2002) is that a child should be asked to label the stimuli, either verbally or manually (e.g., mouse, keypad), by pitch (e.g., high-low-low) or duration (e.g., long-short-long). If this is not possible, the child should be encouraged alternatively to hum or gesture the sound of the stimuli (Musiek et al, 1994; Musiek, 2002; Weihing et al, 2015; Chermak et al, 2017). It may be that labeling the stimuli involves an additional step that adds a greater cognitive demand to the task. Both tasks share several steps including perception, storage in and read out from working memory, and vocalic motor coding. For labeling, an additional step of decision-making, preceding motor coding, may be necessary to categorize the auditory stimuli (e.g., high/low pitch). Alternately, the commonly reported co-occurrence of APD with language impairment (e.g., Ferguson et al,
2011) may limit the ability of those with lesions or delayed development to articulate a verbal response.

Whatever the mechanism, we currently know of no experimental data comparing the influence of response mode on test outcome for the PPS and DPS. The primary aim of this study was to provide those data. If humming is to be used as a substitute for labeling, the results of these two response tasks should be equal. Based on existing reports and the aforementioned analysis, however, we hypothesized that humming would produce superior performance. A secondary aim was to provide additional normative data on both tests.

\section{METHODS}

\section{Participants}

Children were recruited from three primary public schools in the State of São Paulo, Brazil, over 2 years. The children and their parents attended the Clinic School of Speech and Hearing Therapy at Bandeirante University of São Paulo for three assessment sessions, each of 1 hour duration and with a maximum interval of 1 week between the sessions (Table 1). A total 452 children were assessed of whom 201 (44\%; 97 girls and 104 boys) were excluded and 23 (5\%) withdrew. Of the remaining 228 children, 211 (PPS) and 198 (DPS) completed one or both tests. Those not completing both tests had difficulty understanding and/or performing the tasks. Approximately equal numbers of boys and girls of each age contributed data (Figure 1).

At Session 1, parents completed an extensive background questionnaire developed at the Clinic. The 45item questionnaire covered pregnancy history, general health, illnesses, ear problems, developmental and educational history, and current abilities. All children taking the PPS and DPS (see "Procedure") were right handed, aged 7 years to 11 years 11 months (mean age $=9$ years 4 months). Audiometric assessment and PPS and DPS testing were conducted in a sound-attenuated booth. All children had normal hearing defined as pure tone thresholds $\leq 15 \mathrm{~dB}$ HL at octave frequencies $250-8000$ $\mathrm{Hz}$ and tympanograms with peak compensated static acoustic compliance 0.4-1.6 mmhos and tympanometric peak pressure of -100 to $+50 \mathrm{daPa}$ in both ears. They also had $\geq 92 \%$ word recognition performance in each ear (in quiet, $40 \mathrm{~dB}$ above pure tone average threshold at 500,1000 , and $2000 \mathrm{~Hz}$ ). In this test, the children repeated 25 monosyllabic words presented to each ear open-set (Russo and Santos, 2011). Other inclusion criteria for the PPS and DPS testing were that all children 
Table 1. Schedule of Assessment Sessions and Order of Testing

\begin{tabular}{lcc}
\hline First Session & Second Session & Third Session \\
\hline Parent questionnaire & PPS - Humming, & DPS - Pointing, \\
(10) & Right (8)* & Right $(7)^{*}$ \\
General behavior, & PPS - Humming, & DPS - Pointing, \\
speech (15) & Left (6) & Left (5) \\
$\begin{array}{c}\text { Pure tone } \\
\text { audiometry (15) }\end{array}$ & PPS - Labeling, & DPS - Labeling, \\
Rpeech recognition & PPS - Labeling, & DPS - Labeling, \\
quiet (5) & Left (6) & Left (5) \\
Acoustic & & \\
immittance (10) & & \\
Note: Testing order was not counterbalanced. Approximate time \\
taken for each element of the battery is indicated (in minutes). \\
*Including 2-min practice.
\end{tabular}

spoke Portuguese as their first and only language, and had no formal musical training or any known language, learning, neurologic, psychiatric, or behavioral disorder, abnormal pregnancy and/or perinatal history, or delayed neuropsychomotor or otologic history, as determined through the parental questionnaire, completed during the first assessment session (Table 1). Figure 2 shows the proportion of children excluded for each reason.

The same researcher/examiner (first author SAB) performed all these initial screens and all the PPS and DPS evaluations. This researcher is a speech/ language pathologist and audiologist with experience in speech, language, and hearing assessment and central auditory processing assessment. She also developed the instructions, applied the tests, and judged and analyzed the responses. Use of a single researcher reduced variability but did not enable an assessment of interrater reliability.

\section{Procedure}

Both PPS and DPS tests were presented from the Auditec Inc. audio DVD (Child Version, 6-9 years old, 1997) connected to an audiometer (Interacoustic AC40) using calibrated TDH-39 circumaural headphones (ISO-398-1, ISO-398-8). For the PPS (Session 2, Table 1), 30 trials of three consecutive $500 \mathrm{msec}$ duration high $(1430 \mathrm{~Hz})$ or low $(880 \mathrm{~Hz})$ frequency tones with $300 \mathrm{msec}$ interstimulus interval, $10 \mathrm{msec}$ rise-fall time, were presented monaurally at $50 \mathrm{~dB}$ HL. The interval between the trials was $10 \mathrm{sec}$. Six different frequency patterns were presented ten times in random order, first to the right then to the left ear: high-high-low, high-low-low, high-lowhigh, low-high-high, low-high-low, and low-low-high. For the first 30 trials to each ear, the child was asked to hum the sequence and for the next 30 trials, they provided verbal labels (e.g., "high-low-high"). The examiner provided verbal instruction before each set of test trials (humming, label) using, typically, two to three trials (but up to ten trials) repeated by the child to ensure the child understood the task.

DPS (Session 3, Table 1) procedure was nearly identical to PPS. Each trial presented three $1000 \mathrm{~Hz}$ tones of long (500 msec) or short ( $250 \mathrm{msec}$ ) duration. The interval between the tones was $300 \mathrm{msec}$, and the interval between the trials was $6 \mathrm{sec}$. The tones were combined in six different patterns of duration: long-long-short, longshort-short, long-short-long, short-long-long, short-longshort, and short-short-long. Initially, humming was tried as a response mode for DPS, but children appeared to have difficulty with this task, and the examiner also had difficulty judging their responses. For an alternative, nonverbal response, the children instead indicated the duration pattern first by pointing successively at short

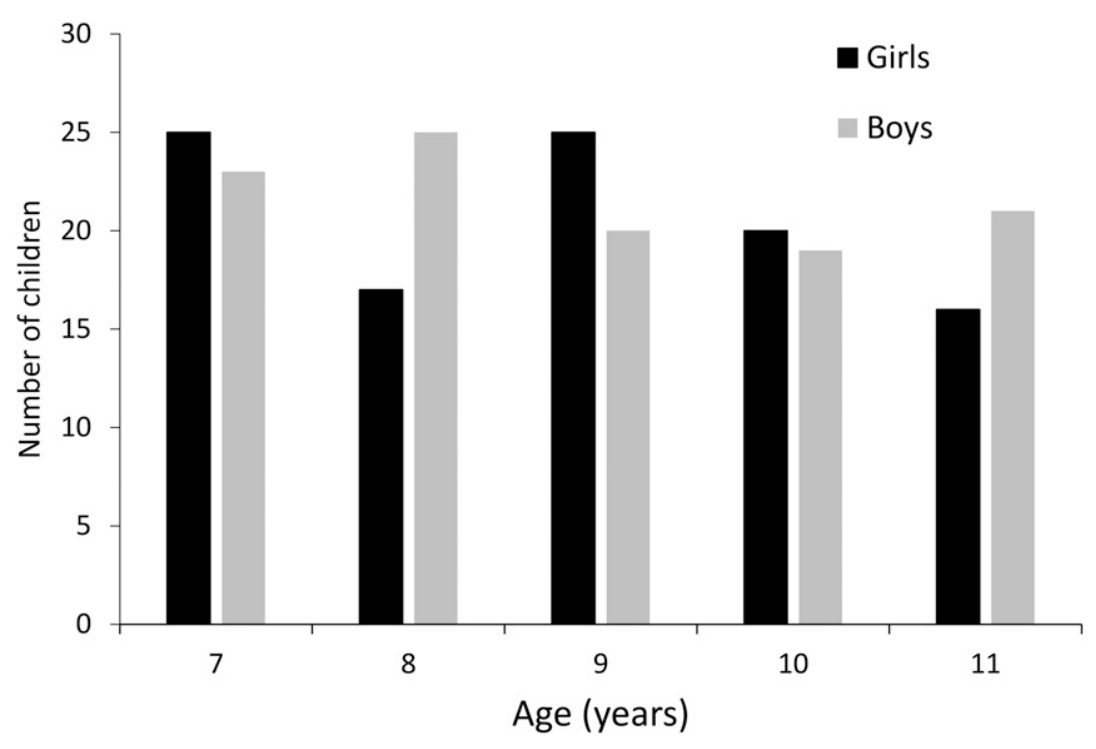

Figure 1. Age and gender distribution of the children tested. 


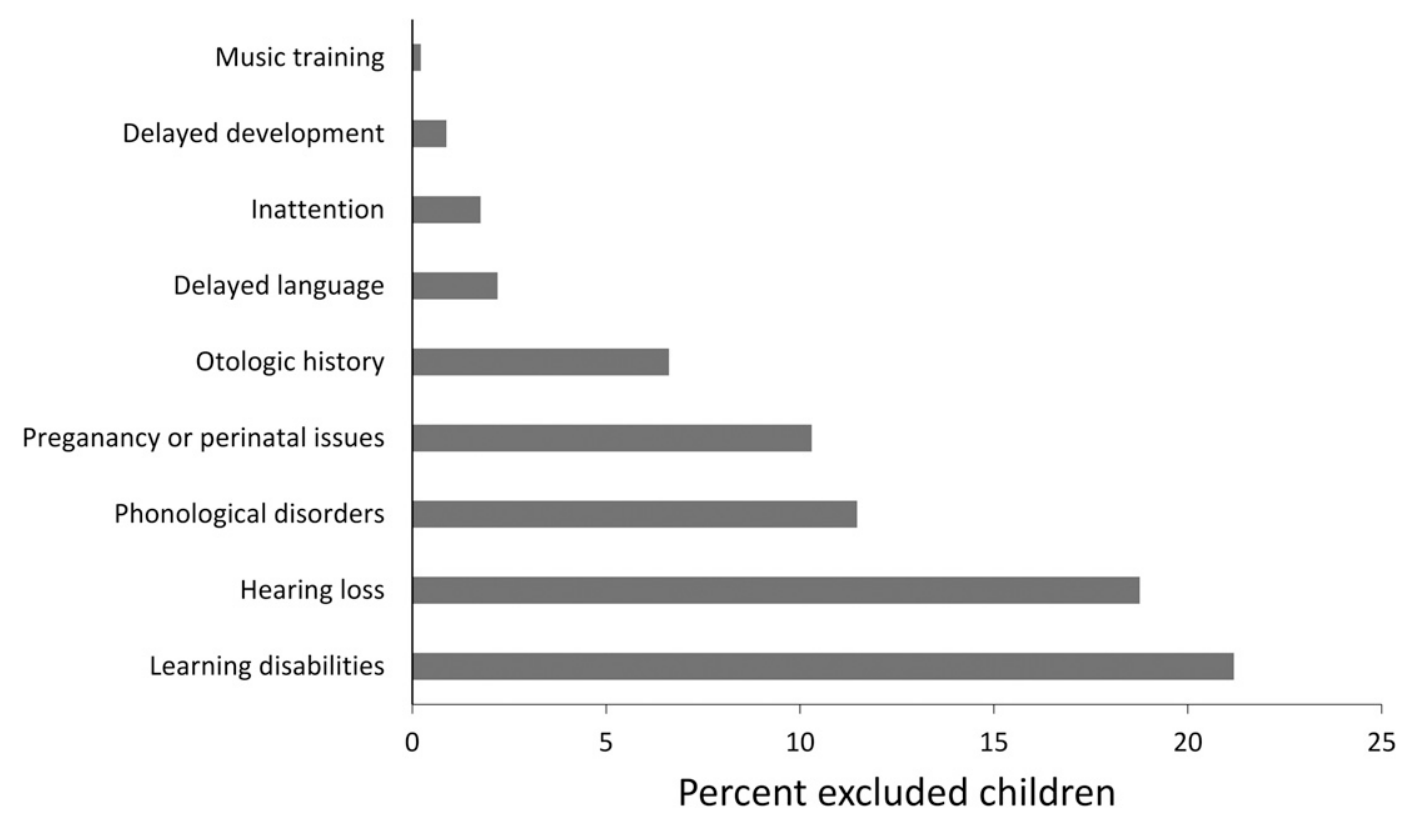

Figure 2. Proportion of children excluded because of each criterion (see "Methods"). The total number of exclusions was 201.

and long objects placed in front of them. After this pointing response, the children provided a verbal label (e.g., "long-short-short"). To explain these tasks, the examiner raised her fingers coinciding with the stimulus order and explained the necessary response. Again, two to three practice trials were typically delivered to confirm understanding of the task.

Order inversion, omission, and insertion of tones in the patterns were common incorrect responses on both tasks (Musiek, 1994) and all response modes. As mentioned earlier, humming responses can be difficult to perceive by the examiner, particularly by an inexperienced examiner and even in the PPS. This point is taken up further in the "Discussion."

The study was approved by the Research Ethics Committee of the Analysis of Research Projects at the Hospital of the Clinics, Medical School, University of São Paulo.

\section{Analysis}

Descriptive statistics were used to summarize sample demographics and outcome measurements. All scores of each child in both tests and ears and all response modes were calculated by the number and percentage of correct answers. The difference between the test scores and ear effect was checked by paired $t$-test or Wilcoxon signed-rank test based on whether the difference distribution was approximately normally distributed.

For PPS humming scores, a two-part model including repeated measures was used to control for a significant "ceiling" effect. In the first part, repeated measures analysis using logistic regression was used to model the probability of a $100 \%$ score and, in the second part, repeated measures analysis using generalized linear mixed models (GLMM) was conducted to predict scores other than full scores (i.e., $<100 \%$ ). A log-normal transformation on the test score was applied in the second part. PPS labeling, DPS pointing, and DPS labeling scores were analyzed by repeated measures analysis using GLMM by PROC GLIMMIX controlling for age and gender. The within-subject factor was ear. The age was centralized at 7 years. The possible interaction effects were also explored in all models.

Spearman rank order correlation was calculated to assess the relationships among the test scores. Data were analyzed using SAS statistical software, version 9.3 (SAS Institute, Cary, NC). A two-sided significance level was set at 0.05 .

\section{RESULTS}

$\mathrm{T}$ he highest scoring test was PPS humming, followed by PPS labeling, DPS pointing and DPS labeling (Figure 3; Table 2). Significant ear differences were identified in all four tests, as indicated by the linear regressions fitted to the data in Figure 3B-D and the mean data in Table 2. For PPS humming (Figure 3A), the left ear score was slightly higher, whereas right ear test scores were substantially higher in the other three tests (PPS labeling, Figure 3B; DPS pointing, Figure 3C; and labeling, Figure 3D). To facilitate comparison across samples and studies, we show in Table 3 median scores and confidence intervals for each test across age and gender.

A major "ceiling" effect was observed in performance on the PPS humming test; 26.5\% (56/211) children scored 30/30 (i.e., 100\%) correct in the right ear humming test and $37.9 \%(80 / 211)$ in the left ear. For twopart modeling (Table 4), results for the first part showed 


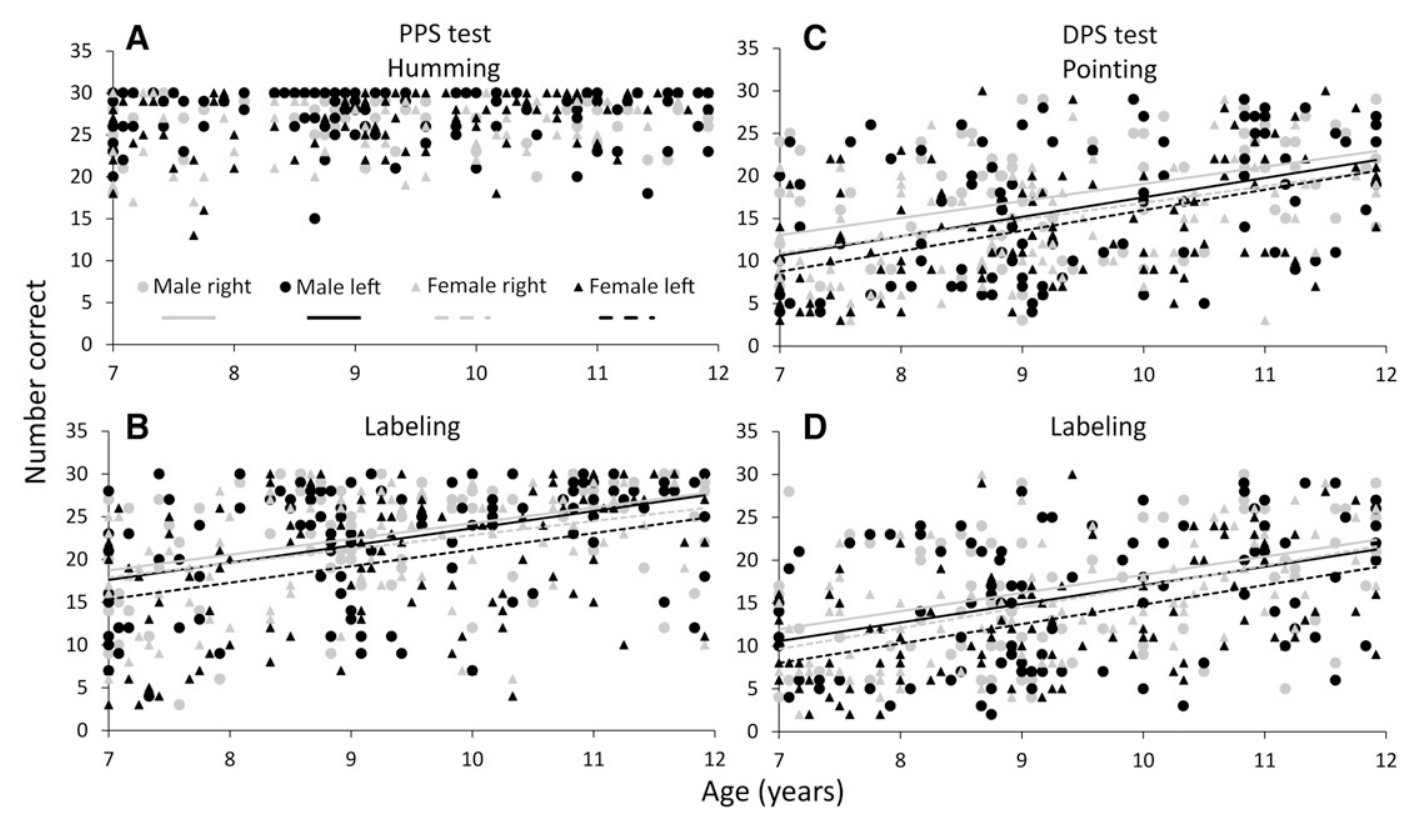

Figure 3. PPS and DPS test results by ear and sex. Number of correct responses for each ear of each girl (female) and boy (male) responding by (A) Humming, (B, D) Labeling, or (C) Pointing. Maximum score for each test was 30. Linear regression lines were fitted to the PPS and DPS data that were symmetrically distributed about the mean.

that the left ear was associated with a significantly higher probability of obtaining a 100\% PPS humming test score than the right ear. In the second part, for scores $<100 \%$, age had a marginally significant, positive linear relationship with log humming test score $(p=0.05)$. No gender difference was found on this test.

Table 2. Sample $(n=228)$ Age, Mean Scores (\% Correct), and Comparison between Tested Ear

\begin{tabular}{lcc}
\hline Variable & & Test Statistics, $p$ Value \\
\hline Age (years), & $9.3(1.5)$ & \\
$\quad$ Mean (SD) & & \\
Range & $7-11.9$ & \\
Male, $\mathrm{n} \%$ & $119(52.2)$ & \\
PPS & $\mathrm{n}=211$ & \\
Humming & Mean (SD) & \\
$\quad$ Right ear & $90.4(10.1)$ & $S=1,013, p=0.038^{\star}$ \\
$\quad$ Left ear & $91.3(10.9)$ & \\
Labeling & & \\
$\quad$ Right ear & $74.6(21.4)$ & $t_{(210)}=4.79, p<0.0001^{\dagger}$ \\
$\quad$ Left ear & $70.4(24.7)$ & \\
DPS & $\mathrm{n}=198$ & \\
Pointing & Mean (SD) & \\
$\quad$ Right ear & $55.5(23.3)$ & $t_{(197)}=4.52, p<0.0001^{\dagger}$ \\
Left ear & $50.6(25.2)$ & \\
Labeling & & \\
$\quad$ Right ear & $53.7(23.4)$ & $t_{(197)}=5.81, p<0.0001^{\dagger}$ \\
Left ear & $48.3(24.7)$ & \\
\hline
\end{tabular}

SD $=$ standard deviation.

*Wilcoxon signed-rank test.

†Paired $t$-test.
For all three remaining tests (PPS labeling, DPS pointing, and DPS labeling), older children had higher scores than younger children, boys had significantly higher scores than girls, and scores were higher in the right than in the left ear (Table 5). For PPS labeling, there was a weak but significant interaction between age and gender, with younger girls performing relatively better than older girls. A significant, positive linear relationship was found between age and test score for each test (Figure 3). Note that the PPS labeling test results also had a marked ceiling effect. However, the GLMM analysis is robust for this variance heterogeneity and nonnormality and showed good fit indices.

All four test scores were significantly, positively related (Figure 4; Table 6). The humming score was only weakly correlated with other tests $(\rho=0.17-0.34)$, likely due to the strong ceiling effect. PPS labeling scores were moderately correlated ( $\rho=0.60-0.68)$ with DPS tests. The two DPS test scores were strongly associated ( $\rho=0.76-0.85$ ). The test scores between the two ears were also strongly correlated, with $\rho$ ranging from 0.72 to 0.85 .

\section{DISCUSSION}

$\mathrm{P}$ erformance on these commonly used PPS and DPS tests varied in a number of ways. Older children performed better than younger children on both tests. Children of all ages performed better on the PPS than on the DPS. They also performed better when asked to hum the tones they had heard than when asked either to point at concrete objects representing the tones or 
Table 3. Median (5\%, 95\%) Scores for Each Test (out of 30) as a Function of Age and Sex (Ears Averaged)

\begin{tabular}{|c|c|c|c|c|}
\hline \multirow[t]{2}{*}{$\overline{\mathrm{Age}}$} & \multicolumn{2}{|c|}{ PPS - Humming } & \multicolumn{2}{|c|}{ DPS - Pointing } \\
\hline & Girls & Boys & Girls & Boys \\
\hline 7 & $27(21,30)$ & $29(20,30)$ & $9(4,22)$ & $14(9,29)$ \\
\hline 8 & $28(17,30)$ & $29(22,30)$ & $10(5,22)$ & $15(8,25)$ \\
\hline 9 & $29(22,30)$ & $28(21,30)$ & $13(7,24)$ & $23(11,29)$ \\
\hline 10 & $28(23,30)$ & $29(24,30)$ & $15(8,22)$ & $22(12,27)$ \\
\hline \multirow[t]{2}{*}{11} & $28(24,30)$ & $28(22,30)$ & $14(5,22)$ & $21(14,28)$ \\
\hline & \multicolumn{2}{|c|}{ PPS - Labeling } & \multicolumn{2}{|c|}{ DPS - Labeling } \\
\hline 7 & $16(7,25)$ & $25(14,29)$ & $9(5,21)$ & $13(6,26)$ \\
\hline 8 & $16(7,26)$ & $23(10,29)$ & $9(5,23)$ & $16(8,25)$ \\
\hline 9 & $25(11,29)$ & $26(15,29)$ & $12(6,23)$ & $23(10,28)$ \\
\hline 10 & $25(18,29)$ & $27(17,30)$ & $14(8,24)$ & $19(11,28)$ \\
\hline 11 & $20(9,27)$ & $28(14,30)$ & $11(5,23)$ & $23(12,28)$ \\
\hline
\end{tabular}

to label the tones verbally. Boys performed better than girls on all tests except the humming test. Stimulation of the right ear gave better results than stimulation of the left ear in three of four tests. Finally, ceiling effects were apparent in the PPS, particularly for older children. Despite these differences, performance of individual children on the two tests was highly correlated.

\section{Sampling and Normalization}

Although this was not intended to be a normalization study, it raised some general questions about recruitment of children for clinical trials of hearing tests and about published normalization values for the PPS and DPS. Because of strict inclusion and exclusion criteria, nearly half the children recruited did not start or complete the test battery. Who is a "typical" child? It depends on the aim of the study. Here, we were primarily interested in comparing performance across tests, stimuli, and response modes, so selecting a relatively able group of children seemed appropriate. However, these children could not be regarded as "typical." For a study focusing on normalization, it is important to recruit across the entire ability range, including population proportionate numbers of children who may be disabled or have other participation difficulties. In this well-powered study, we found some relatively subtle differences that may not have been observed with a smaller or more

Table 4. PPS Humming

\begin{tabular}{lcc}
\hline & Model 1: $100 \%$ & Model 2: $<100 \%$ \\
\hline Age & 0.26 & 0.05 \\
Sex & 0.89 & 0.33 \\
Ear & 0.0004 & 0.57 \\
\hline
\end{tabular}

Note: $p$ values from repeated measures two-part model. Model 1 was a repeated measures analysis using logistic regression to predict the probability of full score (i.e., all 30 correct; 100\%). Model 2 was a repeated measures analysis using GLMM to predict test scores less than $30(<100 \%)$. heterogeneous sample. For example, we found small but highly significant right ear advantages for all tasks except the humming task, where a much smaller left ear advantage, obscured by the ceiling effect, was seen. It appears that previous studies of "frequency pattern tests" (FPTs) have not observed an ear advantage (Weihing et al, 2015). Differences between boys and girls were also seen in three of four tests and these have, likewise, not been previously reported. The number of boys and girls excluded before testing was about equal, so that was not the source of the performance differences reported. We do not know of any other reason why boys performed better.

The large sample used here provided age-related estimates of performance on the PPS and DPS that may have clinical interest. Musiek (2002) and Weihing et al (2015) have presented FPT "norms" of 40\%, 65\%, 72\%, and $75 \%$ based on age $(8,9,10,11+$ years old). FPT stimuli differed from the present study in frequency of the high pitch stimulus (1122 versus $1430 \mathrm{~Hz}$ ), duration (150 versus $500 \mathrm{msec}$ ), and interstimulus interval (200 versus $300 \mathrm{msec}$ ). In each respect, the PPS tests we used should be easier for the children to perform than the FPT tests. Nevertheless, their values are roughly in line with the labeling data here, expressed as percentages (regression line), except at the lower end (mean left and right, 7 , $8,9,10,11$ years old; $54 \%, 77 \%, 73 \%, 78 \%, 84 \%)$. For the humming response, by contrast, our data are more like Musiek's (1994) adult data, "mean" of 88-92\% with little age-related change due to the dominant ceiling effect, also found in another recent, independent, and even larger clinical service study (Moore et al, 2017). For the DPS, equivalent age "norms" for the data presented here were $37 \%, 45 \%, 48 \%, 61 \%$, and $67 \%$. A major reservation concerning the use of these or any other percentage-based norms is that parametric statistics are clearly inappropriate for ceiling results. The PPS humming data, for example, make little sense to report as means and standard deviation (Tables 2 and 3). The other scores were less affected, but the PPS labeling scores of the older children were also hitting ceiling. A detailed critique of percentage scores is provided by Tomlin et al (2014). A further complication is that, according to Tomlin et al (2014), the FPT normalization data are "cutoff scores" rather than a measure of central tendency as used here. Agreed standards for reporting of normalization are much needed.

\section{Maturation}

Almost all studies of the development of hearing have shown superior performance in older than in younger children and almost all those studies have suggested an end point of maturation between 6 and 12 years (Werner, 2007; Moore et al, 2011; Sanes and Woolley, 2011). Analysis of the data shown here suggested that performance on the PPS humming task changed marginally from 7 to 11 years of age. For the other tasks, performance varied 
Table 5. Significant $p$ Values from Repeated Measures GLMM Analysis

\begin{tabular}{lccc}
\hline & PPS Labeling & DPS Pointing & DPS Labeling \\
\hline Age & 0.005 & $<0.0001$ & $<0.0001$ \\
Age * Sex & 0.021 & & \\
Sex & 0.016 & 0.016 & 0.016 \\
Ear & $<0.0001$ & $<0.0001$ & $<0.0001$ \\
\hline
\end{tabular}

substantially between individuals, but there was a relatively steady, linear, and significant increase across this age range, suggesting that further development into adolescence was likely, as shown in a few studies (Huyck and Wright, 2011; Sanes and Woolley, 2011). Generally, our ability to observe maturation depends critically on the task. It is a very demanding requirement for tests such as DPS or FPT with fixed response levels (i.e., nonadaptive) to avoid floor or ceiling effects across a wide range of ages and abilities. This point is discussed further in the following textfor the present study, but a previous study (Moore et al, 2010) examined the maturation of auditory temporal and spectral resolution in a very large, crosssectional sample of 6- to 12-year-old children using two methods. Using one method, they found typical, substantial, and asymptotic maturation when performance was measured on each one of five tests. However, when a different method was used that compared performance between two versions of each task (e.g., the duration of a gap between a tone and a following masking noise), keeping the cognitive demands essentially identical, "no" maturation was seen. Further details of this experiment are provided in the next section and in Moore (2012). This result suggested that measuring sensory maturation using a single behavioral test can produce potentially misleading results. We may assume that the test measures maturation of a sensory attribute (e.g., auditory temporal processing). But this study showed no change with age when nonauditory performance factors were eliminated.

\section{Task Effects}

We found that humming the tone pattern generally produced better performance in the same child than either articulating the attributes of the tones (labeling) or pointing to physical representations of their duration. This suggests that different mechanisms are involved in these responses and that, contrary to current APD recommendations (Musiek, 2002), results from humming should not clinically be combined with or be a substitute for results obtained from the usual labeling task. However, these various response tasks may be useful in further research to identify brain regions involved in normal and impaired auditory processing, for example in future studies using magnetic resonance imaging to help determine the role of various auditory and multimodal cortical areas in processing pattern sequence signals and performing the tests.

It is well understood in psychoacoustics that presenting a multiple choice task with minimal need to understand the properties of the stimulus gives more reliable and better performance. For example, three- or fourinterval forced choice frequency discrimination tasks, asking the listener to pick the odd one out, are preferable to tasks requiring choice of the "higher pitched"

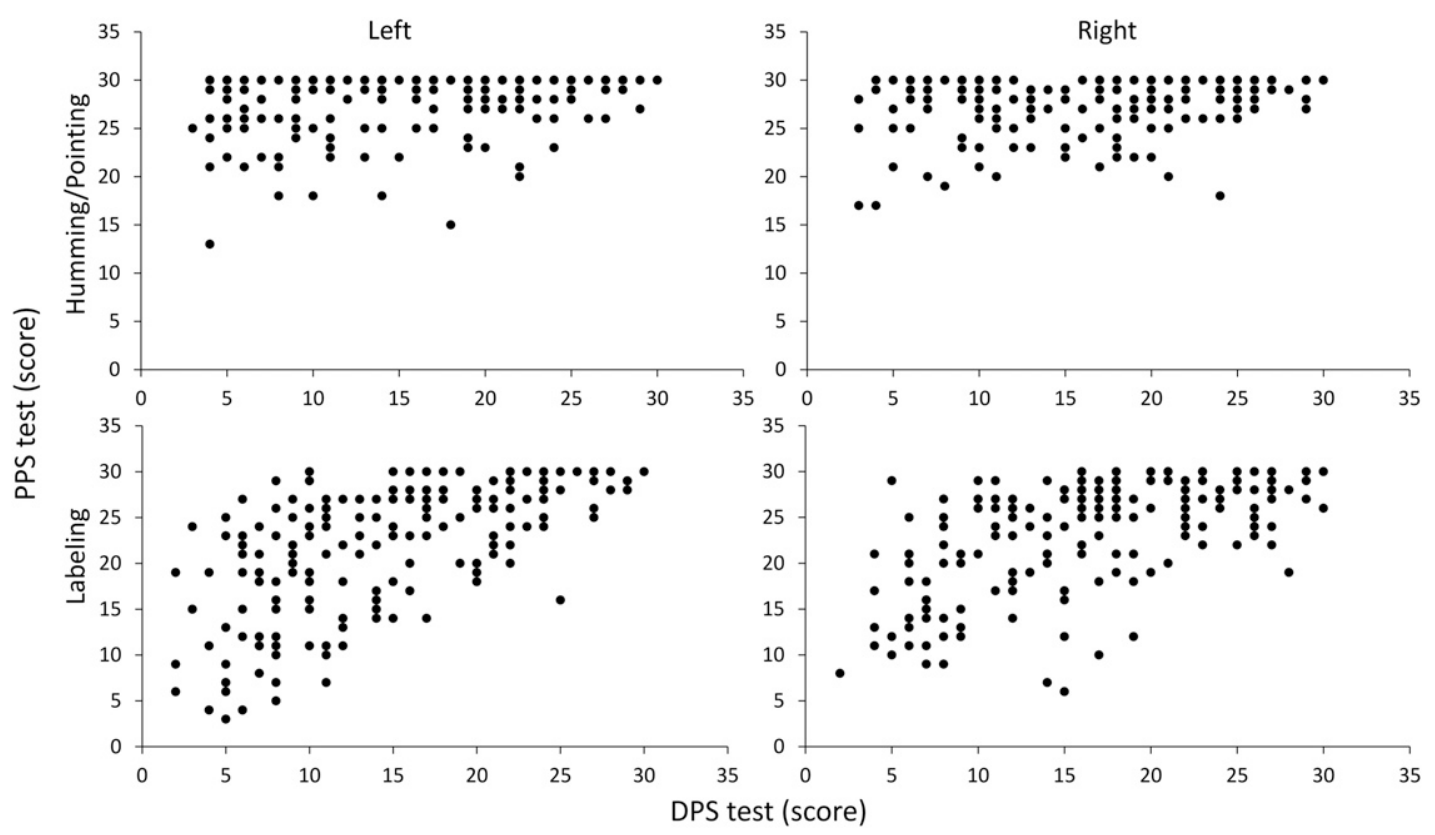

Figure 4. Scatter plots between scores on each test using different response modes (Humming/pointing, Labeling) and different ears (left, right). 
Pitch and Duration Pattern Tests in Children/Balen et al

Table 6. Spearman Rank Order Correlation $(\rho)$ among Test Scores

\begin{tabular}{|c|c|c|c|c|c|c|c|}
\hline & PH_Right & $\overline{\mathrm{PH}} \_$Left & PL_Right & PL_Left & DP_Right & DP_Left & DL_Right \\
\hline$\overline{\mathrm{PH}}$ _Left & 0.72 & & & & & & \\
\hline PL_Right & 0.23 & 0.32 & & & & & \\
\hline PL_Left & 0.26 & 0.34 & 0.84 & & & & \\
\hline DP_Right & 0.20 & 0.22 & 0.60 & 0.61 & & & \\
\hline DP_Left & 0.24 & 0.30 & 0.65 & 0.68 & 0.81 & & \\
\hline DL_Right & 0.17 & 0.25 & 0.60 & 0.62 & 0.80 & 0.83 & \\
\hline DL_Left & 0.18 & 0.27 & 0.66 & 0.67 & 0.76 & 0.85 & 0.85 \\
\hline
\end{tabular}

stimulus (Amitay et al, 2006). To measure task performance, it is also preferable to use adaptive procedures that track the listeners' threshold rather than predetermined step sizes and "percent correct" measures. These procedures give a more unbiased estimate of task performance by avoiding ceiling effects and minimizing the cognitive demands of the task. They are also more efficient because they provide fewer "easy" trials that provide minimal evidence about ability. With current availability of good quality sound delivery via laptop or tablet computers, there is an urgent need to develop future tests for auditory processing using these basic design features. Grube et al (2012) provide an example of how pitch pattern and other auditory sequence tasks may be tested this way. Although humming may be a desirable response output in terms of lack of postperceptual effort, it rests on the ability of the child to accurately reproduce the stimulus and the ability of the tester to interpret the hum.

Pitch and duration pattern tests generally use a labeling response as the default mode of responding (Musiek et al, 2011; Weihing et al, 2015). Although pattern tests may have a place in the assessment of higher level function (ASHA, 2005), tasks that rely on labeling a tonal stimulus should be avoided in testing hearing, at least for children and other special populations such as the elderly or those with known cognitive difficulties. Labeling adds a further level of difficulty to the response that is distinct from the skill to be measured, auditory perception. It is increasingly recognized that cognitive function is inextricably linked with all auditory tasks, even nonspeech tests using the psychoacoustic procedures described previously. For some tests, notably those involving speech perception, decoding and understanding the speech signal is an unavoidable aspect of the task. But for both nonspeech and speech tests, an assessment of auditory processing should aim to minimize attention, memory, language, and learning effects. This can be achieved to a considerable extent by using "derived" or "subtraction" techniques, as described previously (Moore et al, 2010; Moore, 2012) and in other recent papers (Dillon et al, 2014; Cameron et al, 2016), where the cognitive elements are to a large extent cancelled out by subtracting two similar versions of the same test that vary only in the critical auditory element of interest. This design should become a standard element of clinical and research tests.
Studies of patients with lesions of the central auditory nervous system have shown that performance on PPS-like tests is affected in a smaller proportion of patients than is performance on DPS-like tests, leading to a suggestion that these tests involve different functional processes (Musiek et al, 1990). However, we found that performance on the PPS and DPS tests was highly correlated among the large sample of high functioning children examined in this study. The limiting factor for observing this relationship appeared to be a ceiling effect of the PPS test rather than a functional difference between children. It is possible that the previous results reflected the greater difficulty of the DPS together with an overall poorer performance of the patients, resulting in more of them scoring below the "cutoff" ( 2 standard deviation $<$ normal mean).

\section{Study Limitations}

The test order was fixed in this study (Table 1). A preferable design would have been to pseudorandomize the test order between the various conditions in a Latin Square design to ensure each test occurred in each order of presentation. In general, no systematic differences were found between tasks or ears as a function of test order. Right ear performance was generally better than that of left ear but, for the humming task, left ear performance was superior to that of right ear among those scoring $100 \%$. It is possible that this left ear superiority was a learning effect, as right and left ear humming were, respectively, the first and second pattern sequence tests administered. In the third session, performance did not differ significantly between the pointing and labeling tasks (Table 2).

We used the "child" PPS that, as elaborated previously, is easier than the more commonly used FPT (Tomlin et al, 2014). It is therefore likely that the difference in score reported here between the humming and labeling versions of the PPS would be less marked in the FPT, assuming that humming scores would not reach ceiling as readily with the more difficult task.

Finally, the reliability of producing and judging a hummed response is largely uncontrolled. This issue could be addressed to some extent by using two or more "observers" (test staff), but the general point is that 
participant-selected, quantitative measures (e.g., two alternative forced choice; Grube et al, 2012) are preferable to subjective opinion of either the participant or the examiner.

\section{Clinical Implications}

Future use and interpretation of pitch and duration pattern tests should not combine data from humming tones with other forms of response. Because of the ceiling effects in PPS, even in 7-year-olds, it may not be advisable to use the humming response at all. One advisory on this conclusion is, however, that the sample tested here was clearly not representative of the general population. Thus, although some use may be made of these data for normalization purposes, it seems likely that many children who did not pass our stringent inclusion criteria could have brought the mean scores down and simultaneously reduced the ceiling effect, as found in studies of children with impaired reading performing the PPS (Walker et al, 2006) and those using more difficult tone pattern tasks (Tomlin et al, 2014). The age of the child should also be borne in mind when interpreting the results and, for the tasks used in this study, gender and stimulated ear were also important, in contrast to the findings of Willeford (1985) and Musiek (1994).

A final question is whether these and other tests of simple auditory function are useful for diagnosing and managing APD. We live in an acoustically busy and complex world, with sounds coming at us from all directions. Speech is the principal sound we listen to, and that speech is commonly masked by other speakers. Although sound patterns are clearly of importance in perceiving speech, for example, by segregating speech into auditory objects (Bregman, 1990), it seems a big difference between the complexity and rapid modulations of everyday speech and the very slow modulations and spectral purity of the PPS, DPS, and other commonly used tone pattern tests (Emanuel et al, 2011). Further research might focus on the relation between these tests and more functionally meaningful indices of hearing. A recent study that performed such a comparison showed modest but significant correlations between the FPT (Musiek, 1994) and functional benchmarks of listening ability and reading fluency (Tomlin et al, 2015). These correlations were similar to those achieved by the subtests of the Listening in Spatialized Noise-Sentences Test, a speech-in-noise test that has many features of realworld sound listening (Cameron and Dillon, 2007). All auditory processing (AP) test scores were, however, poorer predictors of the benchmark measures than tests of nonverbal IQ and auditory working memory (Tomlin et al, 2015). It may, therefore, be asked whether the weaker correlations obtained with the benchmarks by the AP tests (Tomlin et al, 2015) were due to the auditory or to the cognitive demands of the AP tests.

\section{CONCLUSION}

$\mathrm{A}$ sking children to hum the tone pattern in the PPS produced generally better performance than articulating the attributes of the tones (labeling). In the DPS, pointing to objects did not produce any benefit over labeling. Performance on both tests improved with age. Group performance on the PPS was better than that on the DPS, but individual performance on the two tests was highly correlated.

\section{REFERENCES}

American Academy of Audiology (AAA). (2010) Clinical practice guidelines: diagnosis, treatment and management of children and adults with central auditory processing disorder. https://audiologyweb.s3.amazonaws.com/migrated/CAPD\%20Guidelines\%208-2010. pdf_539952af956c79.73897613.pdf. Accessed November 6, 2017.

Alain C, Woods DL. (1997) Attention modulates auditory pattern memory as indexed by event-related brain potentials. Psychophysiology 34(5):534-546.

Amitay S, Irwin A, Hawkey DJ, Cowan JA, Moore DR. (2006) A comparison of adaptive procedures for rapid and reliable threshold assessment and training in naive listeners. J Acoust Soc Am 119(3):1616-1625.

American Speech-Language-Hearing Association (ASHA). (2005) (Central) Auditory Processing Disorders: The Role of the Audiologist. Rockville, MD: American Speech-Language-Hearing Association.

Bregman AS. (1990) Auditory Scene Analysis: The Perceptual Organization of Sound. Cambridge, MA: The MIT Press.

Cameron S, Dillon H. (2007) Development of the Listening in Spatialized Noise-Sentences Test (LISN-S). Ear Hear 28(2):196-211.

Cameron S, Glyde H, Dillon H, Whitfield J, Seymour J. (2016) The Dichotic Digits difference Test (DDdT): development, normative data, and test-retest reliability studies part 1. J Am Acad Audiol 27(6):458-469.

Chermak GD, Bamiou D-E, Vivian Iliadou V, Musiek FE. (2017) Practical guidelines to minimise language and cognitive confounds in the diagnosis of CAPD: a brief tutorial. Int $J$ Audiol 56(7):499-506.

Dillon H, Cameron S, Tomlin D, Glyde H. (2014) Comments on "Factors influencing tests of auditory processing: a perspective on current issues and relevant concerns" by Tony Cacace and Dennis McFarland. J Am Acad Audiol 25(7):699-703.

Emanuel DC, Ficca KN, Korczak P. (2011) Survey of the diagnosis and management of auditory processing disorder. Am $J$ Audiol 20(1):48-60

Ferguson MA, Hall RL, Riley A, Moore DR. (2011) Communication, listening, cognitive and speech perception skills in children with auditory processing disorder (APD) or Specific Language Impairment (SLI). J Speech Lang Hear Res 54(1):211-227.

Grube M, Kumar S, Cooper FE, Turton S, Griffiths TD. (2012) Auditory sequence analysis and phonological skill. Proc Biol Sci 279(1746):4496-4504.

Huyck JJ, Wright BA. (2011) Late maturation of auditory perceptual learning. Dev Sci 14(3):614-621. 
Kraus N, Chandrasekaran B. (2010) Music training for the development of auditory skills. Nat Rev Neurosci 11(8):599-605.

McDermott EE, Smart JL, Boiano JA, Bragg LE, Colon TN, Hanson EM, Emanuel DC, Kelly AS. (2016) Assessing auditory processing abilities in typically developing school-aged children. $J$ Am Acad Audiol 27(2):72-84.

Moore DR. (2012) Listening difficulties in children: bottom-up and top-down contributions. J Commun Disord 45(6):411-418.

Moore DR, Cowan JA, Riley A, Edmondson-Jones AM, Ferguson MA. (2011) Development of auditory processing in 6- to 11-yr-old children. Ear Hear 32(3):269-285.

Moore DR, Ferguson MA, Edmondson-Jones AM, Ratib S, Riley A. (2010) Nature of auditory processing disorder in children. Pediatrics 126(2):e382-e390.

Moore DR, Sieswerda SL, Grainger MM, Bowling A, Smith N, Perdew A, Eichert S, Alston S, Hilbert LW, Summers L, Lin L, Hunter LL. (2017) Referral and diagnosis of developmental auditory processing disorder (APD) in a large, United States, hospitalbased audiology service. Journal of the American Academy of Audiology DOI: 10.3766/jaaa.16130.

Musiek FE. (1994) Frequency (pitch) and duration pattern tests. J Am Acad Audiol 5(4):265-268.

Musiek FE. (2002) The frequency pattern test: a guide. Hear J 55: 58.

Musiek FE, Baran JA, Pinheiro ML. (1990) Duration pattern recognition in normal subjects and patients with cerebral and cochlear lesions. Audiology 29:304-313.

Musiek FE, Baran JA, Pinheiro ML. (1994) Neuroaudiology: Case Studies. San Diego, CA: Plural Publishing Group.

Musiek FE, Chermak GD, Weihing J, Zappulla M, Nagle S. (2011) Diagnostic accuracy of established central auditory processing test batteries in patients with documented brain lesions. J Am Acad Audiol 22(6):342-358.
Musiek FE, Pinheiro ML. (1987) Frequency patterns in cochlear, brainstem, and cerebral lesions. Audiology 26(6):79-88.

Russo ICAP, Santos TMM. (2011) A Prática da Audiologia Clínica . Sao Paulo, Brazil: Cortez.

Sanes DH, Woolley SM. (2011) A behavioral framework to guide research on central auditory development and plasticity. Neuron 72(6):912-929.

Shinn JB. (2003) Temporal processing: the basics. Hear J 56(7):52.

Skoe E, Krizman J, Spitzer E, Kraus N. (2015) Prior experience biases subcortical sensitivity to sound patterns. J Cogn Neurosci 27:124-140.

Tomlin D, Dillon H, Kelly AS. (2014) Allowing for asymmetric distributions when comparing auditory processing test percentage scores with normative data. J Am Acad Audiol 25(6):541-548.

Tomlin D, Dillon H, Sharma M, Rance G. (2015) The impact of auditory processing and cognitive abilities in children. Ear hear 36(5):527-542.

Walker KM, Hall SE, Klein RM, Phillips DP. (2006) Development of perceptual correlates of reading performance. Brain Res 1124(1):126-141.

Weihing J, Guenette L, Chermak G, Brown M, Ceruti J, Fitzgerald K, Geissler K, Gonzalez J, Brenneman L, Musiek F. (2015) Characteristics of pediatric performance on a test battery commonly used in the diagnosis of central auditory processing disorder. J Am Acad Audiol 26(7):652-669.

Werner LA. (2007) Issues in human auditory development. J Commun Disord 40(4):275-283.

Willeford JA. (1985) Assessment of central auditory disorders in children. In: Pinheiro ML, Musiek FE, eds. Assessment of Central Auditory Dysfunction: Foundations and Clinical Correlates. Baltimore, MD: Williams \& Wilkins.

Zhang Y, Kuhl PK, Imada T, Kotani M, Tohkura Y. (2005) Effects of language experience: neural commitment to language-specific auditory patterns. Neuroimage 26:703-720. 